\title{
Gravitational lensing in the weak field limit by a braneworld black hole
}

\author{
A. S. Majumdar F $^{2}$ and Nupur Mukherjeđ \\ S. N. Bose National Centre for Basic Sciences, Block JD, Salt Lake, Kolkata 700 098, INDIA
}

(Dated: October 30, 2018)

\begin{abstract}
Braneworld black holes existing today may be of primordial origin, or may even be produced in high energy particle collisions in the laboratory and in cosmic ray showers as well. These black holes obey a modified mass-radius relationship compared to standard Schwarzschild black holes. Using the variational principle we calculate the bending angle of a light ray near the horizon of a braneworld black hole in the weak field limit. We next derive the expressions of several lensing quantities like the Einstein radius and the magnification for a point light source. These expressions are modified compared to the lensing quantities for standard Schwarzschild black holes and contain the scale of the extra dimensions.
\end{abstract}

PACS numbers: 11.25.-w, 95.30.Sf, 98.62.Sb

\section{INTRODUCTION}

There is widespread activity in braneworld gravity in recent times [1]. The braneworld scenario of our universe opens up the fascinating possibility of the existence of large extra spatial dimension(s) 2]. The hugely popular Randall-Sundrum (RS-II) braneworld model $[3]$ is made consistent by the requirement that the standard model fields are confined to the brane, except for gravity which could also propagate into the Ads5 bulk which may be of infinite extent but with curvature radius $l$. Current experiments probing the resultant modification of the Newtonian potential constrain the scale of the extra dimension to be $l \leq 0.2 \mathrm{~mm}[4]$.

A specific issue of interest in braneworld gravity is the formation and evolution of black holes $[5]$. Several types of black hole solutions have been obtained in the literature 6, 7]. Black holes formed due to horizon sized density perturbations in the modified braneworld high energy phase of the early universe have a 5 -dimensional Schwarzschild metric. The horizon size of such black holes is proportional to the square root of their mass, a feature that modifies the Hawking temperature, and consequently slows down the evaporation process $[8]$. It has been shown that these black holes accrete radiation in the high energy phase which considerably prolongs their lifetimes [9]. Some of them could survive up to the present era, thus acting as cold dark matter candidates. The braneworld high energy phase is rather conducive to the formation of primordial black hole binaries [10], and gravitational waves from such coalescing binaries are likely to lie within the range of the next generation gravity wave detectors 11]. On the other hand, superhorizon sized black holes which could be formed by various collapse mechanism possess different geometries [] and might evaporate out rapidly as a consequence of AdsCFT correspondence [12].

\footnotetext{
*Electronic address: archan@bose.res.in
}

${ }^{\dagger}$ Electronic address: nupur@bose.res.in
Since the 5-dimensional fundamental scale could be several orders of magnitude below the Planck scale, a lot of current excitement stems from the possibility of braneworld black holes being produced in high energy particle collisions 13. Such a scenario would in principle, open up a direct experimental probe of extra dimensions and (5-d) Planck scale physics. This motivation has led to several specific proposals for black hole formation in $\mathrm{TeV}$ scale dynamics in colliders such as the LHC 14]. Black hole production in cosmic ray showers has also been investigated with discussions on possible signatures 15.

The aim of the present paper is to investigate one potential avenue of observational signatures for extra dimensions. The non-trivial spacetime curvature around the vicinity of black holes could generate interesting motion for massless and massive quanta passing near the horizon. Indeed, the bending of light around standard black holes leads to the resultant observable phenomenon of gravitational lensing which has been widely employed as a mechanism for detecting black holes in astrophysics 16. The analysis of light and particle motion in the context of braneworld gravity is complicated by the absence till date of unique analytical solutions for the metric representing compact objects in higher dimensions. The modification of the standard $1 / r$ form of the Newtonian potential at small distances has nevertheless inspired several attempted solutions [ $[$ based on different physical requirements, and certain corresponding analyses have been performed on the trajectory of light rays and massive particles in such metrics 17, 18]. Some interesting results on orbits around rotating 5 -dimensional black holes have been derived 18]. In this paper we explore the lensing of optical sources by a braneworld black hole in the weak field limit. We first calculate the deflection angle for a light ray passing near the horizon of a braneworld black hole. The corresponding lensing quantities are derived next. Our analysis displays interesting departures of various lensing phenomena and quantities compared to the standard Schwarzschild metric 19].

In our present work we consider a particular suggested geometry used in Refs. [8, 9, 10, 11] describing 
the spacetime metric near the horizon of a 5-dimensional braneworld black hole. Our analysis closely parallels the derivation of the angle of bending of light in the Schwarzschild metric using the variational principle for a null geodesic 20]. Small black holes formed with radius $r_{h} \leq l$ in the early braneworld regime could grow in size by accreting radiation [9, 10]. Even further growth to supermassive dimensions might be possible through accretion of the background "dark" energy 21]. For such black holes the metric far away from the horizon is expected to be of the standard Schwarzschild form [22]. Our interest though is focussed on the region of a few horizon lengths surrounding the black hole where departures from the standard geometry could lead to interesting consequences, and where the deflection of light is accounted for by the weak field limit to a good degree of approximation. The metric in this region is given by [8]

$$
\begin{aligned}
d S_{4}^{2}=-\left(1-\frac{r_{h}^{2}}{r^{2}}\right) & d t^{2}+\left(1-\frac{r_{h}^{2}}{r^{2}}\right)^{-1} d r^{2} \\
& +r^{2}\left(d \theta^{2}+\sin ^{2} \theta d \phi^{2}\right)
\end{aligned}
$$

where the horizon radius $r_{h}$ is related to the black hole mass $M$ by

$$
r_{h}^{2}=\frac{8}{3 \pi}\left(\frac{l}{l_{4}}\right)\left(\frac{M}{M_{4}}\right) l_{4}^{2} \equiv \mathcal{P} M
$$

with $l$ representing the size of the extra dimension, and $l_{4}$ and $M_{4}$ denoting the 4-dimensional Planck length and mass, respectively. Note that Eq.(2) signifies the altered mass-radius relationship for a braneworld black hole (compared to $r_{h}^{\mathrm{S} c h} \sim M / M_{4}$ for the standard Schwarzschild metric) and is at the root of the all the new results for light deviation that follow from the braneworld metric.

We are interested in the equation of motion for a light ray which is described by a null geodesic $d \tau^{2}=$ $g_{i j} d x^{i} d x^{j}=0$. Thus, in terms of an independent affine parameter $\lambda$, for a light ray one could use Eq.(1) and Eq.(2) to obtain

$$
\begin{array}{r}
-\left(1-\frac{\mathcal{P} M}{r^{2}}\right) \dot{t}^{2}+\left(1-\frac{\mathcal{P} M}{r^{2}}\right)^{-1} \dot{r}^{2} \\
+r^{2}\left(\dot{\theta}^{2}+\sin ^{2} \theta \dot{\phi}^{2}\right)=0 .
\end{array}
$$

where we have defined $\frac{d t}{d \lambda}=\dot{t}, \frac{d r}{d \lambda}=\dot{r}, \frac{d \theta}{d \lambda}=\dot{\theta}$ and $\frac{d \phi}{d \lambda}=\dot{\phi}$. Now, using variational principle one obtains the following equations:

$$
\begin{gathered}
\frac{d}{d \lambda}\left[\frac{d t}{d \lambda}\left(1-\frac{\mathcal{P} M}{r^{2}}\right)\right]=0, \\
\frac{d}{d \lambda}\left[r^{2} \sin ^{2} \theta \frac{d \phi}{d \lambda}\right]=0, \\
\frac{d}{d \lambda}\left[r^{2} \frac{d \theta}{d \lambda}\right]=r^{2} \sin \theta \cos \theta\left(\frac{d \phi}{d \lambda}\right)^{2},
\end{gathered}
$$

$$
\begin{array}{r}
\frac{d}{d \lambda}\left[\dot{r}\left(1-\frac{\mathcal{P} M}{r^{2}}\right)^{-1}\right]=-\frac{\mathcal{P} M \dot{t}^{2}}{r^{3}} \\
-\left(1-\frac{\mathcal{P} M}{r^{2}}\right)^{-2} \frac{\mathcal{P} M}{r^{3}} \dot{r}^{2}+r \dot{\theta}^{2}+r \sin ^{2} \theta \dot{\phi}^{2} .
\end{array}
$$

At this stage one could consider without loss of generality the orbit of the light ray to be confined to the equatorial plane, i.e. $\theta=\frac{\pi}{2}$. Using Eqs. (5) and (6) one obtains

$$
r^{2} \dot{\phi}=h
$$

Further, using Eq. (4) one gets

$$
\left(1-\frac{\mathcal{P} M}{r^{2}}\right) \dot{t}=k,
$$

where $h$ and $k$ are constants associated with respectively, the angular momentum and the energy of photons 20]. Substituting Eqs.(8) and (9) in Eq.(3) one gets

$$
\dot{r}^{2}=k^{2}-\left(1-\frac{\mathcal{P} M}{r^{2}}\right) \frac{h^{2}}{r^{2}} .
$$

Now in terms of the variable $u \equiv 1 / r$ Eq.(10) becomes

$$
\frac{d^{2} u}{d \phi^{2}}=-u+2 \mathcal{P} M u^{3}
$$

[The corresponding equation for the Schwarzschild metric is given by $\frac{d^{2} u}{d \phi^{2}}=-u+3 M u^{2} / M_{4}^{2}[20]$. Note that from Eq.(7) one could get an equation in terms of the variable $u$ given by

$$
\frac{d^{2} u}{d \phi^{2}}+\mathcal{P} M u\left(\frac{d u}{d \phi}\right)^{2}+u\left(1-\frac{\mathcal{P} M k^{2}}{h^{2}}\right)=0 .
$$

However, on combining Eq.(12) with Eq.(11) one is led to the equation $\frac{d^{2} u}{d \phi^{2}}+2 u=0$ with no mass term appearing in it. Hence, similar to the case for the standard Schwarzschild metric [20], the $r$-variation equation (7) does not lead to any new input on the calculation of the bending angle. Without the mass term the solution of Eq. (11) is given by $u=u_{0} \cos (\phi)$, with $u_{0}$ representing the inverse of the distance of closest approach. Since the asymptote of the light path is given by $u \equiv 1 / r=0$, the solution $\phi= \pm \frac{\pi}{2}$, signifies no bending of light as is expected to be the case for vanishing mass.

In the weak field limit $\left(r \geq r_{h}\right)$ the r.h.s of Eq.(11) introduces a small deflection of the equation of the light ray from a straight line. The solution of Eq.111 with a small contribution from the mass term can be written as

$$
u=u_{0} \cos (\phi)+\mathcal{P} M u_{0}^{3}\left[\frac{3}{4} \phi \sin (\phi)-\frac{1}{16} \cos (3 \phi)\right] .
$$

For small deflection $\delta$, one can substitute $\phi=\frac{\pi}{2}+\delta$ in Eq. (13) and use the asymptote for the light ray $(u=0)$ to obtain the total bending angle $\alpha=2 \delta$ in the weak field limit given by

$$
\alpha=\frac{3 \pi}{4} \mathcal{P} M u_{0}^{2}
$$


Note that the bending angle for the Schwarzschild metric which could be derived in a similar fashion 20] is given by $\alpha^{\mathrm{Sch}}=4 M u_{0} / M_{4}^{2}$. The ratio of the bending angles which is given by $\alpha / \alpha^{\mathrm{Sch}}=2 l u_{0}$, indicates that the deflection by a braneworld black hole is more prominent for small impact parameters $1 / u_{0}<l$.

The expression for the bending angle of light $\alpha$ derived in the limit of a weak gravitational field follows essentially due the the modified mass-radius relationship for a braneworld black hole (Eq.(2)). Note however, that the bending angle as a function of the impact parameter could also be obtained from the general expression of deflection for a light ray in a spherically symmetric metric 23. This approach was used to calculate the radius of the photon sphere $r_{p}^{\mathrm{Sch}}=3 M / M_{4}^{2}$ and the bending angle in the Schwarzschild metric 24]. A similar analysis can also be performed for braneworld black holes, and it turns out that the the deflection angle can be expanded as

$$
\alpha\left(u_{0}\right)=\frac{3 \pi}{4} \mathcal{P} M u_{0}^{2}\left(1+\frac{1}{2} \mathcal{P} M u_{0}^{2}+\frac{3}{8}(\mathcal{P} M)^{2} u_{0}^{4}+\ldots\right)
$$

for $1 / u_{0}>r_{p}$, with photon sphere defined by $r_{p}=$ $(2 P M)^{1 / 2}$. However, for small impact parameters the weak field approximation loses validity. The discussion of strong gravitational field light bending for Schwarzschild black holes incorporates special features such as the divergence of the deflection angle and the winding number 25. We do not consider such complications in our present analysis, and hence will restrict ourselves to small bending angles given by Eq.(14).

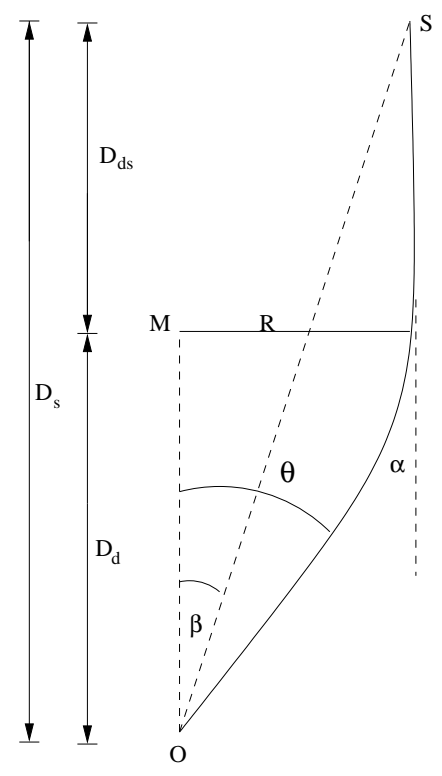

FIG. 1: Gravitationl lensing for point like mass object $M$. A light ray from the source $S$ which passes the lens at a distance $R$ is deflected by $\alpha$. The observer sees an image of the source at angular position $\theta$.
The deflection of light causes the gravitational lensing of a light source located behind the black hole for an observer. We now calculate various lensing quantities like the Einstein angle and the magnification. For pointlike sources, we derive these expressions in an approach that closely parallels the derivation of similar lensing quantities for the standard Schwarzschild metric 19. From Figure 1 it is evident that the condition that the light ray reach the observer is governed by the equation, $\beta D_{s}=\frac{D_{s}}{D_{d}} R-D_{d s} \alpha$. Using the expression for the bending angle in Eq.(14), one gets

$$
\beta=\theta-\frac{3 \pi}{4} \frac{D_{d s}}{D_{s}} \frac{\mathcal{P} M}{\theta^{2} D_{d}^{2}} .
$$

Defining

$$
\alpha_{0}^{2}=\frac{3 \pi}{4} \frac{D_{d s} \mathcal{P} M}{D_{s} D_{d}^{2}}
$$

and

$$
R_{E}=\alpha_{0} D_{d}
$$

where $\alpha_{0}$ and $R_{E}$ are the dimensionless Einstein angle and the Einstein radius [19], respectively, Eq. (16) becomes

$$
\beta=\theta-\frac{\alpha_{0}^{2}}{\theta^{2}} .
$$

This is the lense equation that is obtained for the braneworld metric (11). It is instructive to compare Eq.(19) with the Schwarzschild lens equation which is given by $\beta=\theta-\left(\alpha_{0}^{\text {Sch }}\right)^{2} / \theta$ in which the Einstein angle for the Schwarzschild metric is given by $\alpha_{0}^{\text {Sch }}=$ $\left(\frac{4 M D_{d s}}{M_{4}^{2} D_{d} D_{s}}\right)^{1 / 2}$. The size of the Einstein radius corresponding to the braneworld metric is much smaller compared to the Einstein radius for the same mass in the Schwarzschild metric, i.e.,

$$
\frac{R_{E}}{R_{E}^{\mathrm{Sch}}}=\left(\frac{l}{2 D_{d}}\right)^{1 / 2} .
$$

The image position $\theta$ can be obtained by solving Eq.(19). Note that whereas the Schwarzschild lense equation is quadratic in theta, and hence has two solutions, the braneworld lense equation (19) is cubic in theta, thus having only one real solution for the image position. The real root of Eq. (19) is given by

$$
\begin{aligned}
\theta_{1}=\frac{\beta}{3}+ & \frac{2^{1 / 3} \beta^{2}}{3\left[2 \beta^{3}+27 \alpha_{0}^{2}+3 \sqrt{3} \alpha_{0}\left(\sqrt{4 \beta^{3}+27 \alpha_{0}^{2}}\right)\right]^{1 / 3}} \\
& +\frac{\left[2 \beta^{3}+27 \alpha_{0}^{2}+3 \sqrt{3} \alpha_{0}\left(\sqrt{4 \beta^{3}+27 \alpha_{0}^{2}}\right)\right]^{1 / 3}}{3\left(2^{1 / 3}\right)} .
\end{aligned}
$$

A special case arrises if the lens and the observer are colinear i.e. $\beta=0$ Then (19) becomes

$$
\theta^{3}-\alpha_{0}^{2}=0
$$


whose real solution representing the Einstein ring [19] is given by $\theta=\alpha_{0}^{2 / 3}$.

The magnification $\mu$ produced at the image position $\theta$ is obtained in terms of the source position $\beta$ by the relation [19]

$$
\mu=\frac{\triangle \theta}{\triangle \beta} \frac{\theta}{\beta}=\left|\frac{\theta^{4}}{\theta^{4}-\alpha_{0}^{4}}\right|
$$

From the second equality in the expression for magnification $\mu$ in Eq.(23), one sees that $\mu$ is non-negligible only if $\alpha_{0} \sim \theta$. Using Eqs.(2) and (17), it follows that the impact parameter $R$ should be of the order $R \sim\left[\left(D_{d s} / D_{s}\right)\left(l / l_{4}\right)\left(M / M_{4}\right)\right]^{1 / 2} l_{4}$ for perceptible magnification. The braneworld effect on lensing (i.e., the choice of the metric (1) revealing the 5-dimensional character of gravity at small scales) is feasible for $R \leq l$, implying that $\left(M / M_{4}\right) \leq\left(l / l_{4}\right)$ if $\left(D_{d s} / D_{s}\right) \sim O(1)$. Choosing the value $\left(l / l_{4}\right) \sim 10^{30}$, one obtains the condition $\left(M / M_{4}\right) \leq 10^{30}$, or $M \leq 10^{-8} M_{\text {sun }}$. Black holes with such masses have been classified as sub-lunar compact objects, and standard microlensing results 26] leave open the possiblity of their existence in certain mass ranges as significant fractions of halo dark matter. In the braneworld scenario, primordial black holes with such masses could survive up to the present times 9 . It has been further argued that braneworld black holes in such mass ranges could presently exist in the form of binaries 10] and that gravitational waves emitted during their coalescing stages could be observed in future detectors 11 .

In terms of the qauntities

$$
\begin{aligned}
\beta^{\prime} & =\frac{\beta}{\alpha_{0}} \\
\theta^{\prime} & =\frac{\theta}{\alpha_{0}}
\end{aligned}
$$

and using Eq.(23) the magnification by a braneworld black hole of mass $M$ can be written as

$$
\begin{aligned}
\mu & =\frac{1}{3} \\
+ & \frac{2^{1 / 3} \beta^{\prime}\left[4+\frac{3 \sqrt{3}}{\sqrt{4\left(\beta^{\prime}\right)^{3} \alpha_{0}+27}}\right]}{9\left[2\left(\beta^{\prime}\right)^{3}+\frac{27}{\alpha_{0}}+\frac{3 \sqrt{3}}{\alpha_{0}}\left(\sqrt{4\left(\beta^{\prime}\right)^{3} \alpha_{0}+27}\right)\right]^{1 / 3}} \\
+ & \frac{2^{2 / 3}\left(\beta^{\prime}\right)^{2}\left[3+\frac{3 \sqrt{3}}{\sqrt{4\left(\beta^{\prime}\right)^{3} \alpha_{0}+27}}\right]}{9\left[2\left(\beta^{\prime}\right)^{3}+\frac{27}{\alpha_{0}}+\frac{3 \sqrt{3}}{\alpha_{0}}\left(\sqrt{4\left(\beta^{\prime}\right)^{3} \alpha_{0}+27}\right)\right]^{2 / 3}} \\
- & \frac{22^{4 / 3}\left(\beta^{\prime}\right)^{4}\left[1+\frac{3 \sqrt{3}}{\sqrt{4\left(\beta^{\prime}\right)^{3} \alpha_{0}+27}}\right]}{9\left[2\left(\beta^{\prime}\right)^{3}+\frac{27}{\alpha_{0}}+\frac{3 \sqrt{3}}{\alpha_{0}}\left(\sqrt{4\left(\beta^{\prime}\right)^{3} \alpha_{0}+27}\right)\right]^{4 / 3}} \\
- & \frac{2^{5 / 3}\left(\beta^{\prime}\right)^{5}\left[1+\frac{3 \sqrt{3}}{\sqrt{4\left(\beta^{\prime}\right)^{3} \alpha_{0}+27}}\right]}{9\left[2\left(\beta^{\prime}\right)^{3}+\frac{27}{\alpha_{0}}+\frac{3 \sqrt{3}}{\alpha_{0}}\left(\sqrt{4\left(\beta^{\prime}\right)^{3} \alpha_{0}+27}\right)\right]^{5 / 3}} \\
+ & \frac{\left[2\left(\beta^{\prime}\right)^{3}+\frac{27}{\alpha_{0}}+\frac{3 \sqrt{3}}{\alpha_{0}}\left(\sqrt{4\left(\beta^{\prime}\right)^{3} \alpha_{0}+27}\right)\right]^{1 / 3}}{9\left(2^{1 / 3}\right) \beta^{\prime}} .
\end{aligned}
$$

It can be checked that there is no magnification in the limit of zero mass, i.e., $\mu=1$ when $\beta^{\prime} \rightarrow \infty$. On the other hand, in the limit $\beta^{\prime} \rightarrow 0$, the magnification reduces to

$$
\lim _{\beta^{\prime} \rightarrow 0} \mu=\frac{1}{3}+\frac{1}{3 \beta^{\prime} \alpha_{0}^{1 / 3}} .
$$

Comparison with the magnification produced in the Schwarzschild metric shows that in the $\left(\beta^{\prime} \rightarrow 0\right)$ limit,

$$
\frac{\mu}{\mu^{\mathrm{S} c h}} \approx\left(\frac{M_{4}}{M}\right)^{1 / 6}\left(\frac{l}{l_{4}}\right)^{1 / 3}\left(\frac{l_{4}}{D_{d}}\right)^{1 / 6}\left(\frac{D_{s}}{D_{d s}}\right)^{1 / 6}
$$

Note that a larger size of the extra dimension would produce a brighter image. The magnification produced by such braneworld black hole lenses which could exist in our galactic halo, however, turns out to be diminished compared to the standard Schwarzschild black holes, except for extremely low masses. From Eq.(27), and using the value of $D=D_{d} D_{d} s / D_{s} \sim 10^{22} \mathrm{~cm}$ (relevant for the lensing of galactic halo objects), and $\left(M / M_{4}\right) \sim 10^{30}$, one obtains $\left(\mu / \mu^{\mathrm{Sch}}\right) \sim 10^{-4}$. In fact, one can see from Eq.(27), that in astronomical lensing Schwarzschild black holes would produce brighter images than braneworld black holes for $M / M_{4}>10^{6}$. Thus any braneworld black holes present in the galactic halo would be harder to detect through lensing.

To summarize, in this paper we have investigated weak gravitational lensing of a point-like optical source by a braneworld black hole. The study of particle and light motion in the geometry of higher dimensional black holes is a subject of recent interest 17, 18], particularly so because of the proposed mechanisms of black hole formation in high energy particle collisions and cosmic ray showers $13,14,15]$. Further, as has been shown recently [9], braneworld black holes could survive as relics from the early universe and act as candidates of non-baryonic dark matter. It is feasible for primordial braneworld black holes to exist in the form of binaries [10], and gravitational waves from the coalescence of such binaries could be detected in the near future 11]. Thus, the exploration of the phenomenon of gravitational lensing by braneworld black holes could be of potential utility.

The geometry of a braneworld black hole incorporates a different mass-radius relationship 8 compared to a standard Schwarzschild black hole. In the above analysis, we have calculated the bending angle of light due to the gravitational potential of a braneworld black hole using the variational principle 20]. The consisitency of the derivation through the variational principle is confirmed using the general expression for deflection angle in a spherically symmetric metric 23]. The expression for the bending angle that we have derived contains the scale of the extra dimension $l$. We have next explored the phenomenon of gravitational lensing in the weak field limit. The expressions of lensing quantities like the Einstein angle and the magnification 19] have been calculated in terms of the geometrical parameters and the size 
of the extra dimension. The differences of these expressions from the corresponding ones for Schwarzschild black hole lensing 24 have been highlighted. Further interesting phenomena could be revealed through the analysis of strong gravitational lensing 25] which in the context of the above braneworld geometry has been recently worked out [27]. Strong gravitational lensing in other braneworld metrics have also been studied[28]. Though our present observational capabilities might seem to restrict the status of such analyses to theoretical curiosities, further improvement in techniques might enable the fascinating possiblity of discrimination of different gravity models through observable lensing effects in the not too distant future.
[1] See, R. Maartens, gr-qc/0312059 Living Rev. Rel. 7, 7 (2004) and references therein.

[2] N. Arkani-Hamed, S. Dimopoulos and G. Dvali, Phys. Lett. B429, 263 (1998); I. Antoniadis, N. Arkani-Hamed, S. Dimopoulos and G. Dvali, Phys. Lett. B436, 257 (1998); N. Arkani-Hamed, S. Dimopoulos and G. Dvali, Phys. Rev. D59, 086004 (1999).

[3] L. Randall and R. Sundrum, Phys. Rev. Lett. 83, 4690 (1999).

[4] J. C. Long et al., Nature 421, 922 (2003).

[5] For a recent review, see, P. Kanti, Int. J. Mod. Phys. A19, 4899 (2004).

[6] R. Emparan, G. T. Horowitz, and R. C. Myers, Phys. Rev. Lett. 85, 499 (2000); R. Casadio and B. Harms, Phys. Rev. D64, 024016 (2001); S. Hemming and E. Keski-Vakkuri, Phys. Rev. D64 044006 (2001); R. Casadio, A. Fabbri and L. Mazzacurati, Phys. Rev. D65, 084040 (2002); V. P. Frolov, D. V. Fursaev and D. Stojkovic, JHEP 0406, 057 (2004).

[7] N. Dadhich, R. Maartens, P. Papadopoulos and V. Rezania, Phys. Lett. B487, 1 (2000); M. Bruni, C. Germani and R. Maartens, Phys. Rev. Lett. 87, 231302 (2001); N. Dadhich and S. G. Ghosh, Phys. lett. B518, 1 (2001); S. Shankaranarayanan and N. Dadhich, Int. J. Mod. Phys. D13, 1095 (2004).

[8] R. Guedens, D. Clancy and A. R. Liddle, Phys. Rev. D66, 043513 (2002).

[9] A. S. Majumdar, Phys. Rev. Lett. 90, 031303 (2003); R. Guedens, D. Clancy and A. R. Liddle, Phys. Rev. D66, 083509 (2002).

[10] A. S. Majumdar, A. Mehta and J. M. Luck, Phys. Lett. B607, 219 (2005).

[11] K. T. Inoue and T. Tanaka, Phys. Rev. Lett. 91, 021101 (2003).

[12] T. Tanaka, Prog. Theor. Phys. Suppl. 148, 307 (2003); R. Emparan, A. Fabbri and N. Kaloper, JHEP 0208, 043 (2002); R. Emparan, J. Garcia-Bellido and N. Kaloper, JHEP 0301, 079 (2003).

[13] D. M. Eardley and S. B. Giddings, Phys. Rev. D66, 044011 (2002); K. Cheung, Phys. Rev. Lett. 88, 221602 (2002).

[14] S. Dimopoulos and G. Landsberg, Phys. Rev. Lett. 87, 161602 (2001); L. Anchordoqui and H. Goldberg, Phys. Rev. D67, 064010 (2003); M. Cavaglia, S. Das and
R. Maartens, Class. Quant. Grav. 20, L205 (2003); A. Chamblin, F. Cooper and G. C. Nayak, Phys. Rev. D69, 065010 (2004).

[15] J. L. Feng and A. D. Shapere, Phys. Rev. Lett. 88, 021303 (2002); L. Anchordoqui and H. Goldberg, Phys.Rev. D65, 047502 (2002).

[16] See, for example, EROS collaboration, Astron. Astrophys. 400, 951 (2003); MACHO collaboration, astro-ph/0304464 in "Gravitational Lensing: A Unique Tool For Cosmology", eds. D. Valls-Gabaud and J.-P. Kneib (Aussois, 2003).

[17] M. Visser and D. L. Wiltshire, Phys. Rev. D67, 104004 (2003); S. Kar and M. Sinha, Gen. Rel. Grav. 35, 1775 (2003).

[18] V. Frolov, M. Snajdr and D. Stojkovic, Phys. Rev. D68, 044002 (2003); V. Frolov and D. Stojkovic, Phys. Rev. D68, 064011 (2003).

[19] P. Schneider, J. Ehlers and E. E. Falco, "Gravitational Lenses", (Springer, 1992); F. Bernardeau, [astroph/990117] in "Theoretical and Observational Cosmology", ed. M. Lachieze-Rey (Cargese Summer School, 1998).

[20] See, for example, A. K. Raychaudhuri, S. Banerji and A. Banerjee, "General Relativity, Astrophysics, and Cosmology", Springer-Verlag (New York, 1992).

[21] R. Bean and J. Magueijo, Phys. Rev. D66, 063505 (2002).

[22] R. Emparan, G. T. Horowitz and R. C. Myers, JHEP 01, 007 (2000).

[23] S. Weinberg, "Gravitation and Cosmology: Principles and Applications of the General Theory of Relativity", Wiley (New York, 1972).

[24] K. S. Virbhadra, D. Narasimha and S. M. Chitre, Astron. Astrophys. 337, 1 (1998); K. S. Virbhadra and G. F. R. Ellis, Phys. Rev. D62, 084003 (2000).

[25] V. Bozza, S. Capozziello, G. Iovane and G. Scarpetta, Gen. Rel. Grav. 33, 1535 (2001); V. Bozza, Phys. Rev. D66, 103001 (2002); V. Bozza and L. Mancini, Ap. J 611, 1045 (2004).

[26] C. Alcock et al, Ap. J 499, L9 (1998); G. F. Marani et al, Ap. J 512, L13 (1999).

[27] E. F. Eiroa, gr-qc/0410128

[28] R. Whisker, astro-ph/0411786 